\title{
The global transcriptional regulators, SSN6 and TUP1, play distinct roles in the establishment of a repressive chromatin structure
}

\author{
Julia Promisel Cooper, ${ }^{1}$ Sharon Y. Roth, ${ }^{2}$ and Robert T. Simpson ${ }^{3}$ \\ Laboratory of Cellular and Developmental Biology, National Institute of Diabetes and Digestive and Kidney Diseases, \\ National Institutes of Health, Bethesda, Maryland 20892 USA
}

Repression of a-cell specific gene expression in yeast $\alpha$ cells requires MAT $\alpha 2$ and MCM1, as well as two global repressors, SSN6 and TUP1. Previous studies demonstrated that nucleosomes positioned adjacent to the $\alpha 2 / M C M 1$ operator in $\alpha$ cells directly contribute to repression. To investigate the possibility that SSN6 and TUP1 provide a link between MATa2/MCM1 and neighboring histones, nucleosome locations were examined in ssn6 and tup1 $\alpha$ cells. In both cases, nucleosome positions downstream of the operator were disrupted, and the severity of the disruption correlated with the degree of derepression. Nevertheless, the observed changes in chromatin structure were not dependent on transcription. Our data strongly indicate that SSN6 and TUP1 directly organize repressive regions of chromatin.

[Key Words: $\mathrm{MCM1}$; $\alpha$; chromatin; transcriptional repression; Saccharomyces cerevisiae]

Received March 21, 1994; revised version accepted May 10, 1994.

The nucleosomes that package eukaryotic DNA play integral roles not only as structural elements but also as regulators of gene expression. In many instances, histones have been shown to occupy specific positions with respect to the surrounding DNA sequence, creating what have been called positioned nucleosomes /Grunstein 1990a,b; Wolffe 1990; Simpson 1991). The incorporation of cis-acting regulatory elements into positioned nucleosomes has important implications for the functions of such elements (Simpson 1990); for instance, nucleosomes may prohibit access of transcriptional activating proteins to promoter sequences (Almer and Horz 1986; Almer et al. 1986; Richard-Foy and Hager 1987; Cordingley and Hager 1988; Perlmann and Wrange 1988). Our studies in which nucleosome positioning has been particularly well characterized and associated with transcriptional repression have utilized cell type-specific genes under control of the mating-type system in the yeast Saccharomyces cerevisiae.

Yeast can exist as haploid a or $\alpha$ cells, or as a $/ \alpha$ diploid cells. Mating type is determined by three or four proteins that regulate sets of cell type-specific genes (Herskowitz 1989; Dranginis 1986). The haploid $\alpha$ cell type is speci-

Present addresses: ${ }^{1}$ Department of Chemistry and Biochemistry, University of Colorado, Boulder, Colorado 80309-0215USA; ${ }^{2}$ Department of Biochemistry and Molecular Biology, M.D. Anderson Cancer Center, University of Texas, Houston, Texas 77030 USA.

${ }^{3}$ Corresponding author. fied by two proteins: MAT $\alpha 1$ activates expression of the $\alpha$ cell-specific genes and MAT $\alpha 2$ represses transcription of the a cell-specific genes; neither MAT $\alpha 1$ nor MAT $\alpha 2$ is synthesized in a cells. The MAT $\alpha 2$ repressor contains a region of homology with the Drosophila homeo domain (Laughton and Scott 1984; Hall and Johnson 1987), and like other homeo domain proteins, MAT $\alpha 2$ requires additional proteins to function properly. For example, the binding specificity of MAT $\alpha 2$ is reinforced by its interaction with a non-cell type-specific DNA-binding protein, MCM1; MAT $\alpha 2$ and MCM1 bind cooperatively to a 32 -bp operator located $\sim 200$ bp upstream of the translation initiation codon of each of five known a cellspecific genes (Wilson and Herskowitz 1986; Kronstad et al. 1987; Keleher et al. 1988,1989|. Once bound to the $\alpha 2 / \mathrm{MCM} 1$ operator, MAT $\alpha 2$ requires at least two other proteins, SSN6 and TUP1, for efficient repression of transcription. Keleher et al. (1992) demonstrated that in ssn6 strains, the a cell-specific genes are derepressed, despite the continued binding of MAT $\alpha 2 / M C M 1$ to the $\alpha 2 /$ MCM1 operator; furthermore, when brought to a heterologous promoter via fusion to a LexA bacterial DNAbinding domain, SSN6 acts to repress transcription in a TUP1-dependent manner. Neither SSN6 nor TUP1 has any detectable DNA-binding activity; instead the two proteins are thought to be recruited to the a cell-specific promoter regions by MAT $\alpha 2$ (Keleher et al. 1992). Thus, any model for the mechanism of repression by MAT $\alpha 2$ must incorporate roles for SSN6 and TUP1. 
Previous work from our laboratory has led to the notion that nucleosome positioning is involved in the mechanism of transcriptional repression by MAT $\alpha 2$ (Roth et al. 1990, 1992; Simpson 1990; Shimizu et al. 1991). Comparison of the chromatin structures upstream of an a cell-specific gene in a cells and $\alpha$ cells (in which the MAT $\alpha 2$ repressor is absent and present, respectively) indicated that binding of MAT $\alpha 2$ to its operator leads to the very precise placement of an array of nucleosomes adjacent to the $\alpha 2 / \mathrm{MCM} 1$ operator; this nucleosomal array is absent in a cells (Roth et al. 1990; Shimizu et al. 1991). The most proximal nucleosome in this array incorporates essential promoter elements for a cell-specific gene expression, the TATA box being located near the nucleosomal pseudodyad, thus suggesting that steric hindrance by the nucleosome might contribute to the MAT $\alpha 2$-mediated repression (Shimizu et al. 1991). Additional strong support for this latter idea stems from the fact that yeast $\alpha$ cells harboring mutations in the aminoterminal tail of histone $\mathrm{H} 4$ display partial derepression of a cell-specific transcription. This derepression is accompanied by destabilization of nucleosomes or altered nucleosome locations near the $\alpha 2 / M C M 1$ operator (Roth et al. 1992). Finally, the ability of MAT $\alpha 2$ to position nucleosomes adjacent to its operator in numerous heterologous contexts suggests that positioning occurs via an active mechanism, rather than being a by-product of the absence of transcription (Simpson 1990, 1993; Roth et al. 1990; Morse et al. 1992).

The current work aims to synthesize the observed requirements for SSN6 and TUPl with the evidence that nucleosomes are involved in repression by MAT $\alpha 2$. Both ssn6 and tup1 mutations have pleiotropic effects, including flocculence and derepression of the glucose-repressible genes in addition to the mating type defects (Carlson et al. 1984; Trumbly 1986; Schultz and Carlson 1987; Mukai et al. 1991); effects on chromatin structure could provide a unifying mechanism for these diverse phenotypes. Both SSN6 and TUP1 are homologous to proteins associated with other systems. SSN6 contains 10 repeats of the tetratricopeptide (TPR) domain, a putative protein-protein interaction domain that has been found in proteins associated with a number of processes, including mitosis, transcriptional regulation, RNA splicing, protein import, and neurogenesis (Sikorski et al. 1990; Goebl and Yanagida 1991). The TPR domains are essential for SSN6 function (Schultz et al. 1990). TUP1 contains six repeats of a 43-amino-acid domain that was first found in the $\beta$-subunit of the G-protein, transducin (Fong et al. 1986); removal of a single G-protein repeat abolishes TUP1 activity (Williams and Trumbly 1990). SSN6 and TUP1 have been shown to exist in a complex; intriguingly, several of the TPR proteins have been associated functionally with members of the $\beta$-subunit G-protein family (Goebl and Yanagida 1991). Here, we show that deletion of either SSN6 or TUP1 in $\alpha$ cells leads to the disruption of nucleosome positioning adjacent to the $\alpha 2$ / MCMI operator, thus lending support to the model in which local nucleosome positioning or an organized chromatin domain plays a key role in the mechanism of repression by MAT $\alpha 2$. We show further that the tup 1 mutation affects chromatin structure more severely than does the ssn 6 mutation, correlating with the relative severities of the two mutations with regard to their effects on gene expression. Finally, we show that the effects on nucleosome positioning are unlikely to be secondary to the transcriptional state of the underlying DNA.

\section{Results}

Previous work has established that the MAT $\alpha 2$ repressor precisely positions an array of nucleosomes adjacent to its operator and has suggested that this nucleosome positioning plays an important role in the mechanism of transcriptional repression by MAT $\alpha 2$ (Shimizu et al. 1991; Roth et al. 1992). Because SSN6 and TUP1 are required for full repression of a cell-specific gene expression (Keleher et al. 1992), we reasoned that these proteins might be involved in MAT $\alpha 2$-mediated nucleosome positioning. To explore this possibility, we deleted most of the SSN6- and TUP1-coding sequences individually in yeast $\alpha$ cells and examined the consequences of the gene disruptions for chromatin structure and expression of MAT $\alpha 2$-regulated genes.

\section{High-resolution micrococcal nuclease mapping of the promoter region for an a cell-specific gene in ssn 6 and tup $1 \alpha$ cells}

Figure 1 shows a genomic primer extension map of micrococcal nuclease cleavage sites in the promoter region and coding sequence for STE6, an a cell-specific gene. As observed in previous experiments, the wild-type $\alpha$-cell chromatin exhibits a characteristic pattern of protection from micrococcal nuclease cleavage relative to the cleavage pattern for naked DNA (Shimizu et al. 1991). Regions of protection from nuclease cleavage that encompass $\sim 150 \mathrm{bp}$ and indicate translationally positioned nucleosomes are separated by nuclease hypersensitive sites that correspond to linker regions; an array of such positioned nucleosomes is observed extending into the coding sequence for STE6 (the top of the footprint). This pattern has led to the assignment of nucleosome positions shown by ellipses at the right of Figure 1 (Shimizu et al. 1991). In wild-type a cells, which do not express the MAT $\alpha 2$ protein, sequences throughout the region are cleaved frequently by micrococcal nuclease, indicating the absence of stable nucleosome positions. In addition, unique nuclease hypersensitive sites are observed in a-cell chromatin. Note in particular that increased accessibility to micrococcal nuclease is observed for sequences that map to the TATA box in a-cell chromatin; these same sequences are protected from nuclease cleavage in $\alpha$ cells.

In ssn 6 and tup $1 \alpha$ cells, nucleosome positioning in the STE6 promoter region is markedly disrupted. Numerous cleavage sites are observed in the region downstream of the $\alpha 2 / \mathrm{MCM} 1$ operator, including sites that incorporate the TATA box for STE6. Furthermore, the array of nu- 


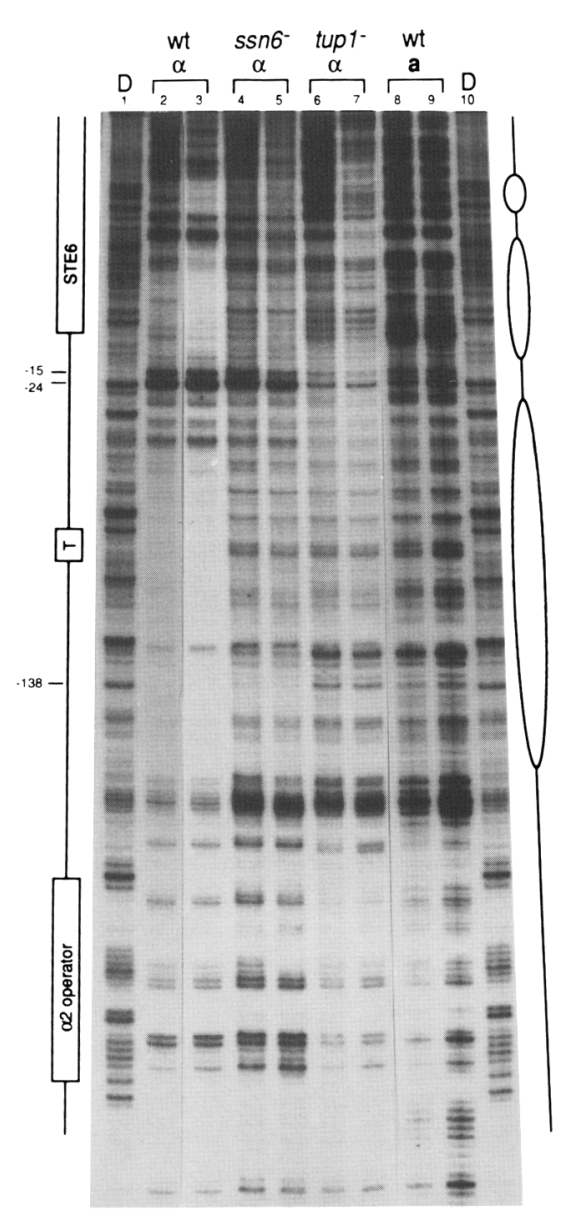

Figure 1. Chromatin structure of the STE6 gene in wild-type cells and ssn 6 and tup1 mutants. Micrococcal nuclease cleavage sites downstream of the $\alpha 2 / \mathrm{MCM} 1$ operator at the genomic STE6 locus were mapped by primer extension. (D; lanes 1,10 ) indicates the naked (protein stripped) DNA samples that control for the sequence specificity of micrococcal nuclease. The remaining lanes $(2-9)$ represent micrococcal nuclease digestion of chromatin at two nuclease levels for each strain (see Materials and methods|, with the cell types specified above. Locations of the $\alpha 2 / \mathrm{MCM}$ operator, consensus TATA box (T), and STE6coding sequence are indicated to the left. Inferred locations of positioned nucleosomes in the wild-type $\alpha$ cells are indicated by the ellipses to the right.

cleosomes extending into the STE6-coding sequence in wild-type $\alpha$ cells appears to be absent in the ssn 6 and tup1 mutants. Interestingly, discrete differences exist between the cleavage patterns of the two mutants. The nuclease hypersensitive sites that are observed in wildtype $\alpha$ cells at positions -15 and -24 are present in the ssn6 mutant but not in the tup1 mutant, and a hypersensitive site that maps to position -138 in a-cell chromatin is observed in the tup1 a cell but not its $\operatorname{ssn} 6$ counterpart. In both instances, the cleavage pattern obtained from tup1 cells resembles more closely that obtained from a cells than does the ssn 6 cleavage pattern, which retains more of the characteristics of the wildtype $\alpha$ cell. Thus, the tup 1 deletion appears to have more severe consequences with regard to genomic chromatin structure than the $\operatorname{ssn} 6$ deletion.

\section{Alteration of chromatin structure of a plasmid containing the $\alpha 2 / M C M 1$ operator in $\operatorname{ssn} 6$ and tupl $\alpha$ cells}

The MAT $\alpha 2$ repressor has been shown to position nucleosomes adjacent to its operator not only at its natural chromosomal locations but also in numerous heterologous contexts (Roth et al. 1990; Simpson 1990, 1993; Morse et al. 1992). An example of this is provided by the TALS minichromosome (Fig. 2), a TRP1/ARS1 derivative containing the STE6-derived $\alpha 2 / \mathrm{MCM} 1$ operator (Roth et al. 1990). The TATA box normally used in STE6 transcription is absent from this operator construct. Figure 2 shows an indirect end label map of micrococcal nuclease cleavage sites in TALS chromatin. Minichromosomes isolated from wild-type $\alpha$ cells exhibit a ladder of discrete cut sites, most of which are not observed in the naked DNA control. The sites are separated by regions of $\sim 150 \mathrm{bp}$ of protection from nuclease cleavage; this pattern indicates an array of positioned nucleosomes extending around the plasmid (Roth et al. 1990; Fig. 2). In wild-type a cells, this pattern is disrupted in the region adjoining the $\alpha 2 / \mathrm{MCMl}$ operator; the hypersensitive

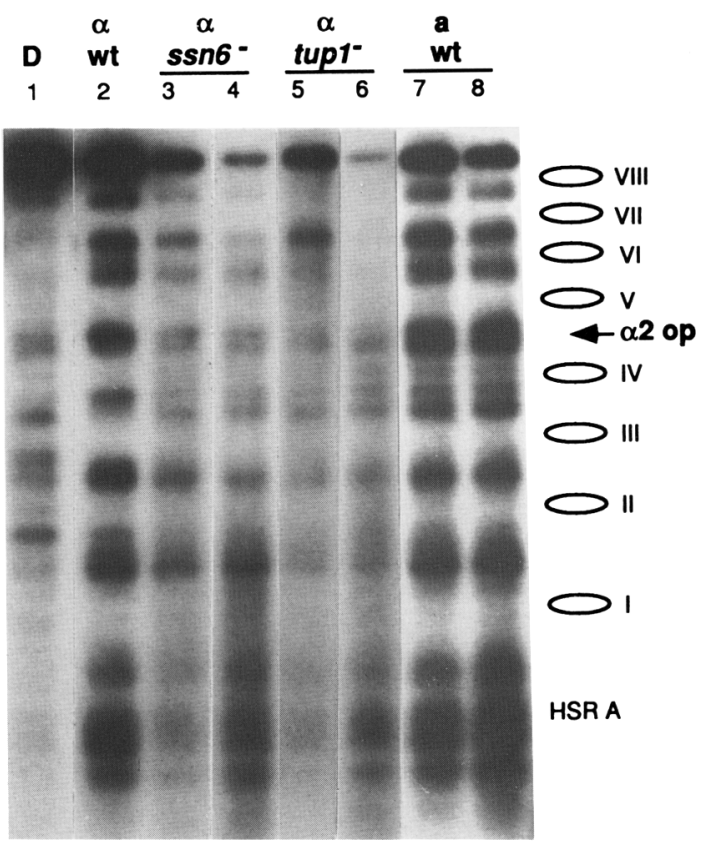

Figure 2. Indirect end-label map of micrococcal nuclease cleavage sites in plasmid chromatin. The TALS plasmid, which contains an $\alpha 2 / \mathrm{MCMl}$ operator, was mapped in a clockwise direction from the EcoRV site. (D; lane 1) indicates the naked DNA control for the sequence specificity of micrococcal nuclease. The remaining lanes $(2-8)$ represent micrococcal nuclease digestion of plasmid chromatin at various nuclease levels (see Materials and methods); cell types are indicated above. Inferred locations of positioned nucleosomes in the wild-type $\alpha$ cell are indicated by the ellipses to the right. 
sites at the $\alpha 2$ operator and between nucleosomes III and IV in $\alpha$ cells are shifted to smaller sizes in the absence of the MAT $\alpha 2$ protein, and the nucleosome IV region itself is rendered accessible to micrococcal nuclease cleavage. Thus, stable nucleosome positioning in this region requires the MAT $\alpha 2$ protein. In both ssn 6 and tup1 mutant $\alpha$ cells, the TALS footprints are less well defined than those obtained from wild-type $\alpha$ cells, possibly signaling some destabilization of plasmid nucleosome positioning in the mutant strains. Disruption of nucleosome positioning adjacent to the $\alpha 2 / \mathrm{MCM} 1$ operator is clear; there is nuclease cutting in the region occupied in wild-type $\alpha$ cells by nucleosome IV, and the hypersensitive sites between nucleosomes III and IV and at the $\alpha 2$ operator are shifted and broadened. The digestion pattern near the operator is nearly identical for the two mutant strains and a cells. Therefore, the SSN6 and TUP1 proteins are required for nucleosome positioning by the MAT $\alpha 2$ repressor in the minichromosome context as well as in the genomic context.

\section{Analysis of plasmid topoisomer distributions in the ssn 6 and tupl backgrounds}

Earlier studies have established that the TALS minichromosome isolated from a cells contains one less negative supercoil than the $\alpha$ cell-derived plasmid (Roth et al. 1990, 1992). Because each nucleosome imparts one negative supercoil to a plasmid, one possible interpretation of this result is the presence of one more nucleosome on the TALS plasmid in $\alpha$ cells relative to a cells. We therefore performed chloroquine gel analyses of plasmid topoisomer distributions for the ssn 6 and tup1 mutant strains, as shown in Figure 3. The positions of the centers of each Gaussian distribution of topoisomer bands (Fig. 3, dots) indicate the linking numbers of the TALS plasmid in the four strains (Morse 1991). The superhelical density of TALS isolated from $\operatorname{ssn} 6 \alpha$ cells is only slightly reduced (by $<1$ negative supercoil) from that of the minichromosome obtained from wild-type $\alpha$ cells. In marked contrast, the tup1 background results in the loss of almost two negative supercoils from the TALS plasmid relative to TALS from wild-type $\alpha$ cells. The increased severity of the tup 1 deletion relative to the ssn 6 deletion with regard to plasmid supercoiling parallels the relative severities of the two mutations with regard to nucleosome positioning in the genomic context.

The extent of the tup1-linking number deficit was unexpected because it exceeds that of TALS derived from a cells. One explanation for such a phenomenon would invoke an effect of the TUP1 protein that is independent of the MAT $\alpha 2$ repressor; perhaps TUP1 plays a role both in general nucleosome assembly and in the assembly of the specific chromatin structure that is directed by the MAT $\alpha 2$ repressor. However, when topoisomer distributions are compared for the TRP1/ARS1 plasmid, which lacks an $\alpha 2 /$ MCM1 operator (Roth et al. 1990), no differences in linking number are observed between the various strains; all are within 0.2 linking number (Fig. 3). The effects of SSN6 and TUP1 on superhelical density
TALS

\section{TRP1/ARS1}

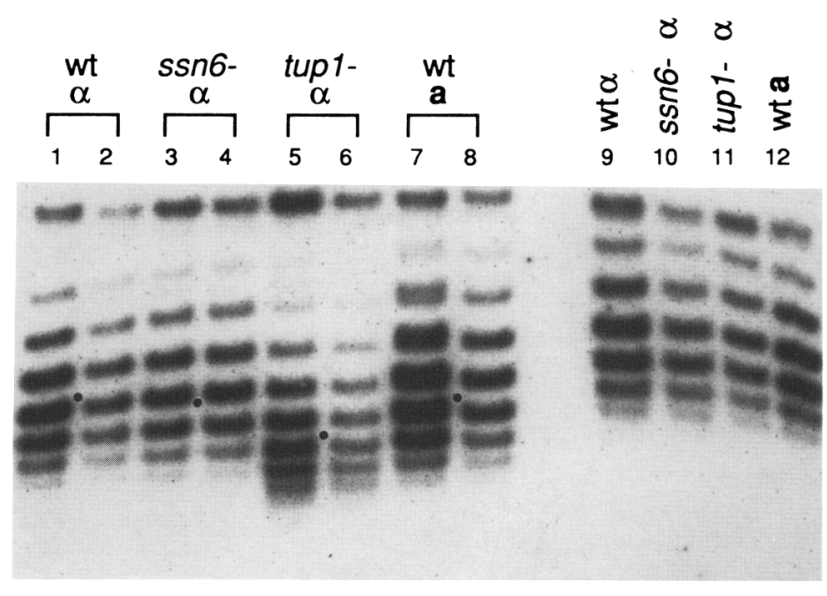

Figure 3. Topoisomer distributions of the TALS and TRP1/ ARS1 plasmids. Plasmid supercoil density was analyzed by chloroquine gel electrophoresis. Under the conditions employed (see Materials and methods), the faster migrating bands represent the least negatively supercoiled species. Plasmid and cell types are indicated above; for the TALS plasmid, two independent isolates of each cell type are shown. The black dots indicate the centers of the Gaussian distributions of topoisomers for each cell type, determined as described in Morse (1991).

are therefore specific to $\alpha 2 / \mathrm{MCM} 1$ operator-containing plasmids.

\section{Comparison of the relative effects of $\operatorname{ssn} 6$ and tup 1 deletions on a cell-specific gene expression}

The differences in chromatin structures observed in the ssn 6 and tup1 mutants suggest that the two proteins play discrete roles in setting up the MAT $\alpha 2$-directed chromatin domain. To determine whether the ssn 6 and tup1 mutations also exhibit differential effects on MAT $\alpha 2$ regulated gene expression, we performed experiments that examine the expression of MAT $\alpha 2$-controlled genes. Yeast a cells produce a-factor, a pheromone that arrests $\alpha$ cells in the $G_{1}$ phase of the cell cycle; $a$-factor production requires expression of $M f a 1$ and $M f a 2$, both of which are transcriptionally repressed in $\alpha$ cells by MAT $\alpha 2$. To assess qualitatively whether $M f \mathbf{a} 1$ and $M f \mathbf{a} 2$ are derepressed in ssn 6 and tup $1 \alpha$ cells, we monitored a-factor production by performing a halo assay /Chan and Otte 1982, Fig. 4). In this assay, strains to be tested for a-factor production are streaked onto a lawn of $\alpha$ cells that are supersensitive to a-factor ( $\alpha$ sst 2 cells); a zone of growth inhibition (halo) in the $\alpha$ sst 2 lawn surrounding a tested colony indicates that the colony has secreted a-factor. As expected, wild-type $\alpha$ cells do not produce a-factor and therefore do not elicit a halo in the surrounding lawn of $\alpha$ sst 2 cells, whereas wild-type a cells do produce a-factor and consequently do generate a zone of growth inhibition. The $\operatorname{ssn} 6 \alpha$ and tup $1 \alpha$ strains both generate a-factor halos; therefore, both mutations lead to derepression of 
Figure 4. Halo assay for a-factor production. Cells to be tested for a-factor secretion were streaked over lawns of $\alpha$ sst2 cells (see Materials and methods). Cell types are indicated; a zone of growth inhibition (halo) surrounding a colony indicates production of a-factor. The two plates represent identical experiments with two independent sets of isolates.

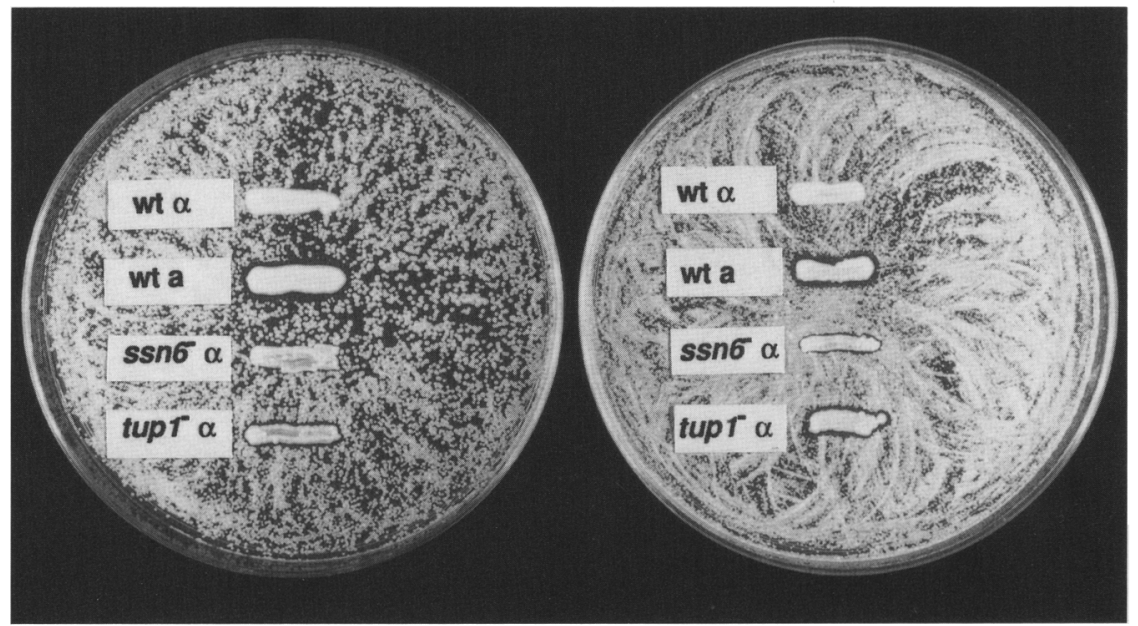

the a cell-specific genes. However, the extent of derepression differs between the two strains: The tup $1 \alpha$ cells reproducibly elicit larger halos than do the $\operatorname{ssn} 6 \alpha$ cells.

To quantify differences in the extent of derepression of a cell-specific gene expression between the ssn 6 and tup1 strains, we disrupted the two genes in cells containing a lacZ reporter gene fused to the genomic STE6 promoter (kindly provided by R.H. Morse, NIH, Bethesda, MD) and measured $\beta$-galactosidase activities in cell extracts (Rose and Botstein 1983; Rose et al. 1990; activities were normalized to the amount of protein assayed because the flocculent phenotypes of the mutants preclude accurate optical density measurements). As shown in Figure 5, expression from STE6 is repressed $\sim 30$-fold in $\alpha$ cells relative to a cells. A modest reversal of this repression $(\sim 2.5$-fold $)$ is observed in $\operatorname{ssn} 6 \alpha$ cells; in contrast, the

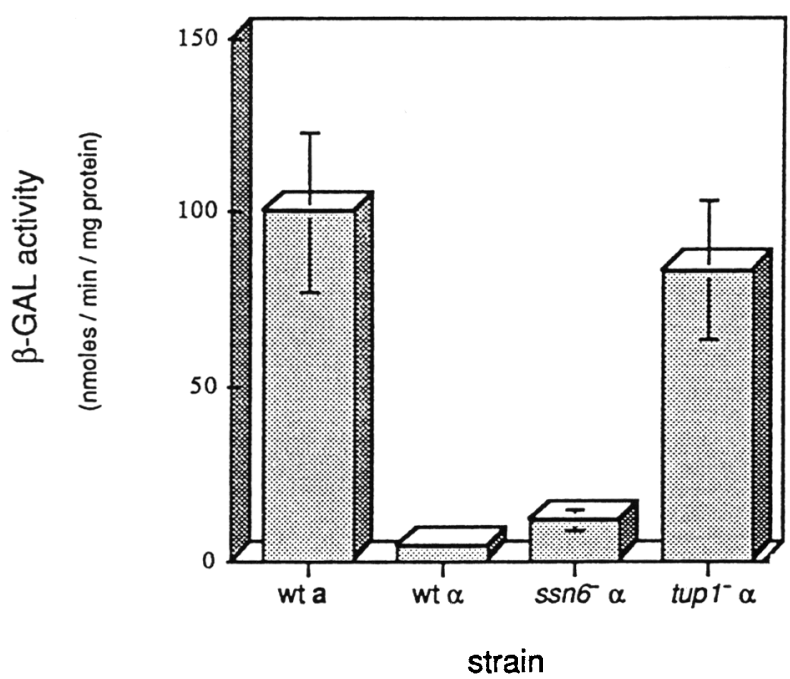

Figure 5. Activities of STE6-lacZ fusions. $\beta$-Galactosidase assays were performed as described previously (Rose and Botstein 1983; Rose et al. 1990) on cells containing a lacZ reporter fused to the genomic STE6 promoter. $\beta$-Galactosidase activities were normalized to the amount of protein assayed. tup $1 \alpha$ cells exhibit a 25 -fold derepression of STE6-lacZ with $\beta$-galactosidase activity reaching $85 \%$ of that observed in a cells. Clearly, the tup 1 phenotype is more severe than the ssn 6 phenotype not only with regard to effects on chromatin organization but also with regard to derepression of a cell-specific transcription.

Comparison of STE 6 chromatin structures in $\mathbf{a}, \alpha$, and tupl $\alpha$ cells in which STE6 transcription has been nearly eliminated

The foregoing data support the possibility that nucleosome positioning constitutes part of the mechanism of transcriptional repression by MAT $\alpha 2$, SSN6, and TUP1. However, the data do not distinguish whether the observed differences in chromatin structure of the STE6 gene are a direct consequence of MAT $\alpha 2 / S S N 6 / T U P 1$ action or, instead, stem secondarily from differences in transcriptional activity. To address this latter issue, we sought to eliminate transcription in a cells, $\alpha$ cells, and tup $1 \alpha$ cells and determine whether the differences in nucleosome organization between the three strains would be retained. We therefore produced strains harboring a number of point mutations in the STE6 TATA box and other STE6 promoter elements (another potential TATA box and two of the pheromone response elements; Fig. 6). RNase protection assays performed in the a-cell derivatives showed conclusively that STE6 transcription was reduced to $5-7 \%$ of the wild-type levels.

Figure 6 shows a genomic primer extension map of micrococcal nuclease cleavage sites in the mutated STE6 promoter regions. The mutant $\alpha$ cell pattern (Fig. 6, lanes $1,2)$ is virtually identical to that obtained from wild-type $\alpha$ cells (Fig. 1, lanes 2,3), with clearly defined regions of protection from nuclease cleavage of $\sim 150 \mathrm{bp}$ separated by nuclease hypersensitive sites, indicating an array of positioned nucleosomes extending downstream from the $\alpha 2 / \mathrm{MCM} 1$ operator. The pattern obtained from a cells harboring the STE6 promoter mutations (Fig. 6, lanes 5,6) again closely resembles that of the wild-type a cells (Fig. 1 , lanes 8,91 . Numerous nuclease cleavage sites are ob- 


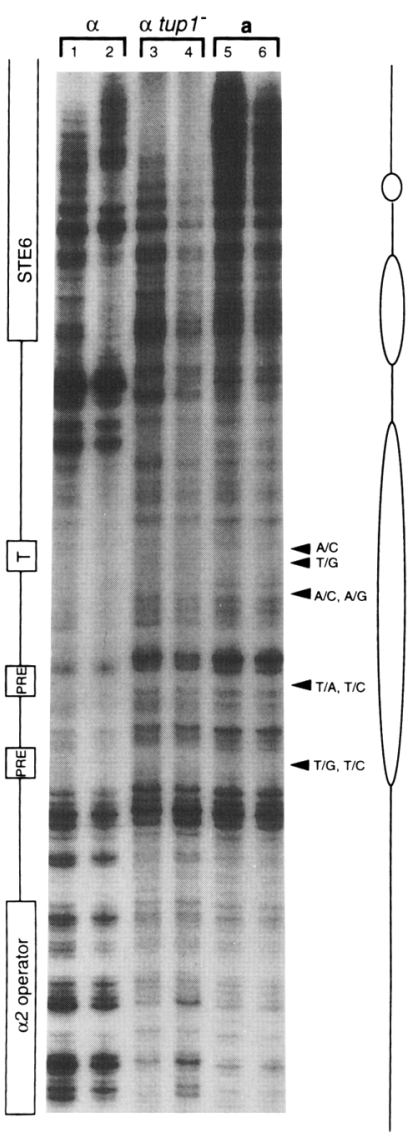

Figure 6. Chromatin structure of the STE6 gene in cells containing alterations in the STE6 promoter. Micrococcal nuclease cleavage sites were mapped by primer extension in cells containing point mutations in STE6 promoter elements. Locations of the point mutations are indicated by arrowheads (right); for each mutation, the wild-type and mutated residue are indicated at left and right, respectively, of / symbols for each arrowhead. Locations of the $\alpha 2 / \mathrm{MCM} 1$ operator, the mutated consensus TATA box $(T)$, pheromone response elements $\{\mathrm{PRE}\}$, and the STE6-coding region are shown at the left of the figure. Cell types are specified above the gel; for each cell type, two levels of micrococcal nuclease digestion are shown (see Materials and methods). Inferred locations of positioned nucleosomes in the $\alpha$ cell are indicated by the ellipses at right.

served in the regions corresponding to positioned nucleosomes in $\alpha$ cells, thereby indicating that even when STE6 transcription is drastically reduced, nucleosome positioning in this region does not occur in cells lacking the MAT $\alpha 2$ protein. It is interesting to note the only difference in the cleavage patterns obtained from wildtype a cells and promoter-mutant a cells: The nuclease hypersensitive site that occurs at the TATA box in wildtype a cells is missing in the TATA mutants. As was the case for the wild-type STE6 promoter, the pattern obtained from promoter-mutant tup1 $\alpha$ cells is similar to the a-cell pattern. The protected regions observed in the TUP1 $\alpha$ cells that correspond to positioned nucleosomes are not observed, and the hypersensitive site at the TATA box constitutes the only prominent difference between the wild-type and mutant STE6 promoters in the tup1 background. Thus the tup1 mutation leads to the disruption of nucleosome positioning in $\alpha$ cells in a manner that appears to be independent of STE6 transcription.

\section{Discussion}

The maintenance of mating type identity in yeast $\alpha$ cells requires the general transcriptional repressors SSN6 and TUP1 (Keleher et al. 1992). In the current work, we have delineated one of the means whereby SSN6 and TUP1 exert their effects; both proteins appear to play key roles in setting up a repressive chromatin structure. Our results have important implications with regard to the mechanism of repression of a cell-specific genes.

\section{Model for repression of a cell-specific genes by the MAT 2 protein}

The pronounced effects of SSN6 and TUP1 disruptions on nucleosome positioning by the MAT $\alpha 2$ repressor lend strong support to a model that invokes chromatin organization as part of the mechanism of MAT $\alpha 2$ action (Roth et al. 1992), as summarized schematically in Figure 7. As described above, previous studies have established that binding of MAT $\alpha 2$ leads to an array of positioned nucleosomes adjacent to the $\alpha 2 / \mathrm{MCM} 1$ operator (Roth et al. 1990, 1992; Shimizu et al. 1991). Aminoterminal mutations in histone $\mathrm{H} 4$ result in disruption of this nucleosome positioning, to an extent that correlates tightly with the extent of derepression of a cell-specific transcription in the H4 mutants (Roth et al. 1992). Deletion of either SSN6 or TUP1 leads to the loss of nucle-

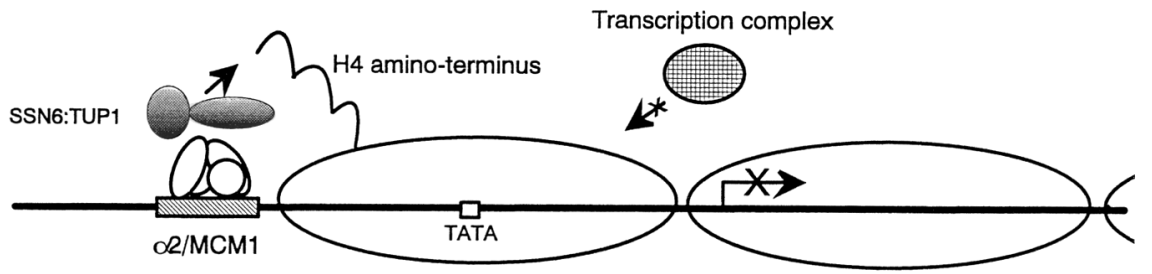
tion of downstream nucleosomes in a process that requires the amino-terminal sequences of histone H4 (Roth et al. 1992). The size and stoichiometry of the SSN6/TUP1 complex are not known. The organized chromatin structure contributes to repression by occluding transcription factors from promoter elements.
Figure 7. Model for MAT $\alpha 2$-mediated transcriptional repression. The promoter region for an a-cell specific gene is shown with an array of nucleosomes (large ellipses) positioned downstream of the $\alpha 2$ / MCM1 operator. Binding of MAT $\alpha 2 /$ MCM1 leads to the recruitment of SSN6 and TUP1, which then facilitate organiza- 
osome positioning adjacent to an $\alpha 2 / \mathrm{MCM} 1$ operator in $\alpha$ cells, both in the context of the genomic MAT $\alpha 2$-regulated STE6 gene and in the context of a plasmid-borne $\alpha 2 / M C M 1$ operator. Taken together, our data suggest that the SSN6 and TUP1 proteins constitute at least part of a link between the MAT $\alpha 2$ repressor and the aminoterminal tail of histone $\mathrm{H} 4$. It should be noted that whereas the $\mathrm{H} 4$ mutants and the ssn 6 strains exhibit similar degrees of chromatin disruption and transcriptional derepression of MAT $\alpha 2$-regulated genes, the $\mathrm{H} 4$ mutants do not display the clumpy phenotype that appears to be diagnostic of reduced SSN6 expression. Therefore, we view as unlikely the possibility that the effects of histone $\mathrm{H} 4$ mutations in the $\alpha 2$ system stem indirectly from effects on transcription of SSN6 and TUP1.

Additional powerful evidence in support of the mediation of nucleosomes in MAT $\alpha 2$ transcriptional repression is provided by our data showing that the STE6 chromatin structures found in a, $\alpha$, and tup $1 \alpha$ cells persist when STE6 transcription is nearly eliminated via point mutations of the STE6 TATA boxes and pheromone response elements (PREs). It should be noted that a small amount of residual STE6 transcription is retained in the promoter-mutant a cells $(<7 \%$ of the wild-type level). Therefore, we cannot unconditionally rule out the possibility that this low level of transcription is sufficient to confer the virtually complete disruption of chromatin structure that we observe in the STE6 promoter-mutant a cell, although we find this latter scenario to be highly implausible. Transcription levels are lower from the mutant promoter in a and tup1 $\alpha$ cells than from the wildtype promoter in $\operatorname{ssn} 6 \alpha$ cells, yet the disruption of nucleosome positioning is more severe in a and tup $1 \alpha$ cells than in ssn $6 \alpha$ cells. Furthermore, chromatin structure is disrupted around the $\alpha 2 / \mathrm{MCM} 1$ operator when in a minichromosome, removed from its normal TATA box. Thus, the data strongly suggest that the absence of positioned nucleosomes in the STE6 promoter region in a cells and tup $1 \alpha$ cells is not a consequence of transcriptional activity but, instead, stems directly from the lack of the MAT $\alpha 2$ (in a cells) or TUP1 (in tup1 $\alpha$ cells) proteins.

The phenomenon of chromatin effects on transcription must be viewed in terms of hierarchies of proteinprotein and protein-DNA interactions, with some chromatin structures being more resistant to transcriptional activation than others and some proteins being more capable than others of overcoming the repressive effects of nucleosomes. Thus, the chromatin domain that is organized by the MAT 22 repressor is not dominant over all transcriptional activators. Morse et al. (1992) demonstrated that the cis-acting elements essential for transcription of the RNA polymerase III-transcribed sup4-o tRNA $^{\text {Tyr }}$ gene could function when placed downstream of an $\alpha 2 /$ MCM1 operator. Morse (1993) also showed that GAL4, a transcriptional activator of genes transcribed by RNA polymerase II, is able to disrupt nucleosomes that are positioned by MAT $\alpha 2$. In addition, Herschbach and Johnson (1993) found that MATa2 could repress tran- scription of RNA polymerase I-transcribed genes but not of genes that are transcribed by RNA polymerase III. Although these latter results were interpreted to reflect interactions between MAT $\alpha 2$ (and associated proteins) and specific transcriptional subunits shared by RNA polymerases I and II but not III, they are also consistent with numerous examples of changes in chromatin structure associated with activation of polymerase I- and IItranscribed genes (Prior et al. 1983; Conconi et al. 1989; Wolffe 1992). In contrast, highly active tRNA genes appear to be resistant to the formation of most repressive chromatin structures (Morse et al. 1992). The foregoing examples illustrate that some transcriptional activators can overcome the MAT $\alpha 2$-generated chromatin domain, whereas others cannot. Likewise, for some systems, a round of DNA replication may be required to reorganize repressive chromatin into an active conformation, whereas other systems may employ proteins that can interact with nucleosomal DNA (Schmid et al. 1992). In the case of the MAT $\alpha 2$-repressed genes, transcriptional activation would occur only following mating-type switching (Herskowitz 1989) and therefore would not be likely to occur without an intervening round of DNA replication.

In addition to SSN6 and TUP1, other proteins are likely to be involved in nucleosome positioning by the $M A T \alpha 2$ repressor. Candidate proteins include SIN3, SIN4, and RPD3, which are all required for full repression of a cell-specific transcription in $\alpha$ cells /Vidal et al. 1991; Vidal and Gaber 1991; Jiang and Stillman 1992; Chen et al. 1993). The large size (1200 kD) of the SSN6/ TUP1 complex determined from immunoblotting experiments (Williams et al. 1991) suggests that other proteins may associate with SSN6 and TUP1. The roles of the above factors in the organization of chromatin are currently under investigation.

\section{Repression of other genes by SSN6 and TUP1}

The involvement of nucleosomes in SSN6/TUP1 action may extend to other genes for which the two proteins have been identified as negative regulators, the most prominent examples being the glucose-repressible genes (Carlson et al. 1984; Schultz and Carlson 1987). Recent studies of the glucose-repressed gene SUC2 suggest a situation that is analogous in some ways to the regulation of a cell-specific genes. The DNA-binding protein MIG1 may act to recruit SSN6 and TUP1 to the SUC2 promoter (Nehlin et al. 1991), where both may participate in nucleosome positioning. In a study by Matallana et al. (1992), the investigators interpreted their data to indicate the presence of two positioned nucleosomes in the SUC2 promoter region when cells were grown in media containing glucose (the repressed state), although this interpretation was complicated by a paucity of cut sites for naked DNA in the region occupied by putative nucleosomes. In ssn 6 mutants, which are nonrepressible, an open chromatin structure was observed even in the presence of glucose (Matallana et al. 1992). Hirschorn et al. (1992) further showed that specific factors, including 
SNF2/SWI2 and SNF5, are required both for SUC2 transcription and for the exclusion of nucleosomes from the SUC2 promoter. The effects of SNF2/SWI2 and SNF5 on chromatin in the SUC2 promoter region are unaffected by mutation of the SUC2 TATA box and therefore are not secondary to SUC2 transcription (Hirschorn et al. 1992). Thus, SNF2/SWI2 and SNF5 appear to function by overcoming the repression conferred by particular chromatin structures. This idea is supported by the finding that mutations in histone $\mathrm{H} 3$ (SIN2) suppress mutations in SNF2/SWI2 (Prelich and Winston 1993).

An interesting activity of the SSN6 protein has been reported recently by McDonnell et al. (1992). By use of a reconstituted estrogen-responsive transcription system in yeast, those investigators found that SSN6 acts as a repressor of transcription by the TAF1 domain of the estrogen receptor. Although a model in which mutation of SSN6 leads to global alterations in chromatin structure was disfavored by McDonnell et al. (1992), the possibility remains that such mutations lead to changes in the chromatin structures of a subset of genes to which the SSN6 protein is specifically recruited. Thus, if a mammalian counterpart to the SSN6 protein exists, it will be worthwhile to investigate the possibility that such a protein acts in part via regulation of chromatin structure in mammalian cells.

\section{The SSN6 and TUP1 proteins play distinct roles in the $\alpha 2$ system}

The tup1 phenotype is more severe with regard to its effects on nucleosome positioning adjacent to the $\alpha 2 /$ MCM1 operator than the ssn6 phenotype (see Results). Furthermore, the tup 1 mutation leads to a 10 -fold greater derepression of a MAT $\alpha 2$-controlled reporter gene than the ssn 6 mutation. The correlation between the differential effects of the two mutations on levels of a-specific gene repression and their relative effects on chromatin structure provides further support for the contention that repression and chromatin structure are closely associated. Moreover, the foregoing results suggest that TUP1 plays a more dominant role than SSN6 in MAT $\alpha 2$ repression.

The most dramatic difference between ssn 6 and tup1 phenotypes occurs in their relative effects on plasmid topoisomer distributions. The ssn6 disruption leads to only a modest loss of supercoiling relative to the wildtype $\alpha$ cell, suggesting that whereas the ssn 6 defect results in disruption of nucleosome positioning, it does not lead to the loss of a nucleosome from the TALS plasmid. In contrast, the tup1 disruption leads to the loss of approximately two negative supercoils from TALS relative to TALS isolated from wild-type $\alpha$ cells; it is possible that this result reflects the loss of approximately two nucleosomes from TALS chromatin in the tup1 strain. The lack of any impact of the tup1 mutation on TRP1/ ARS1 supercoiling indicates that the tup1 effect is specific to plasmids that contain an $\alpha 2 / M C M 1$ operator, that is, the tup1 mutation does not lead to a general disruption of chromatin. We have observed that the
TALS topoisomer distribution in tup1 a cells is also reduced (by $\leqslant 1$ negative supercoil) relative to wild-type a cells (J.P. Cooper, unpubl.), suggesting that MAT $\alpha 2$ is not required for all of the tup1 effects on plasmid supercoiling. It is therefore conceivable that TUP1 is associated with MCMI in the plasmid context. The identification of both TUP1 and MCM1 in screens for autonomously replicating sequence (ARS)-specific plasmid maintenance (Thrash-Bingham and Fangman 1989; Passmore et al. 1989/ may hint at such an association.

The functions of the TPR domain found in SSN6 and the G-protein domain found in TUP1 have yet to be defined rigorously. Although the SSN6 protein does not appear to cofractionate with putative nuclear scaffold components (Williams et al. 1991), the occurrence of other TPR proteins in this fraction (Goebl and Yanagida 1991) might suggest an association, which could provide a focal point for the regulation of several genes by a macromolecular complex. Further study of SSN6 and TUP1 may yield insights into the relationships between nuclear organization, chromatin assembly, and transcriptional regulation.

\section{Materials and methods}

Yeast strains, plasmids, and media

S. cerevisiae strains FY23 (MATa ura3-52 trp1 $\Delta 63$ leu2d1) and FY24 (MAT $\alpha$ ura3-52 trp1 $\Delta 63$ leu2 $\Delta 1$ ) were grown according to standard procedures (Rose et al. 1990) in either rich media (YEPD) or selective media (SDM or SCM supplemented with the appropriate amino acids). 5-FOA plates were prepared as described (Rose et al. 1990). Plasmids pRS406 (Sikorski and Hieter 1989), TALS (Roth et al. 1990), and TRP1/ARS1 (Thoma et al. 1984) have been described previously. Yeast transformations were performed according to the DMSO-enhanced transformation protocol of Hill et al. (1991).

\section{Disruption of the SSN6 and TUP1 genes}

Gene disruptions were effected by $\gamma$ - or $\Omega$-transformation utilizing the yeast integrating plasmid pRS406 /Sikorski and Hieter 1989 |. With these protocols, sequences flanking a region of the genome that is to be deleted are cloned in tandem into a vector containing a selectable marker but no origin for replication in yeast. Following transformation of yeast with the aforementioned vector, recombination between the flanking sequences on the vector and in the genome leads to replacement of the region between the flanking sequences with vector sequences. To disrupt SSN6, DNA fragments corresponding to base pairs 243-432 and 1907-2133 of the SSN6 sequence (with base pair 1 denoting the A residue of the translational start site; Schultz and Carlson 1987) were produced by PCR of yeast genomic DNA and cloned in tandem into the pRS406 polylinker region; the resultant construct was linearized between the two SSN6 segments and used to transform FY24 cells to uracil prototrophy. Thus, base pairs 432-1907 of SSN6 were replaced with pRS406 sequences. TUP1 was disrupted in a similar fashion, by use of fragments corresponding to base pairs 86-254 and 17531964 of the TUP1 sequence /with base pair 1 denoting the A residue of the translational start site; Williams and Trumbly 1991; Zhang et al. 1991), resulting in replacement of base pairs 254-1964 of the TUP1 gene with pRS406 sequences. Both ssn6 and tup 1 mutants displayed clumpy phenotypes; in addition, 
both disruptions were verified by genomic Southern blotting (Southern 1975).

Mutation of TATA boxes and PREs in

the STE6 promoter region

Yeast strains harboring point mutations in the consensus TATA box and one other potential TATA box, as well as two PREs upstream of the genomic STE6 sequence, were produced using a pop-in/pop-out allele replacement strategy (Rothstein 1991). A fragment encompassing -679 to +157 of the STE6 sequence (with position 1 denoting the A residue of the translational start site), in which several point mutations were introduced, was produced by PCR techniques using as a template the pUCSTE6 plasmid (McGrath and Varshavsky 1989). The point mutations were the following: $-157, \mathrm{~T} \rightarrow \mathrm{C} ;-156, \mathrm{~T} \rightarrow \mathrm{G} ;-140, \mathrm{~T} \rightarrow \mathrm{C}$; $-139, \mathrm{~T} \rightarrow \mathrm{A} ;-115, \mathrm{~A} \rightarrow \mathrm{G}_{;}-114, \mathrm{~A} \rightarrow \mathrm{C} ;-103, \mathrm{~T} \rightarrow \mathrm{G}_{;}-100$, $\mathrm{A} \rightarrow \mathrm{C}$; the point mutations created novel PstI and $B c$ II sites for subsequent verification by Southern blot analysis. The fragment was cloned into the integrating vector pRS406 (Sikorski and Hieter 1989/, which contains a URA3 marker. Following linearization of the resulting clone at the $K p n I$ site at +97 of STE6, the clone was used to transform FY23 (MATa) and FY24 (MAT $\alpha$ ) cells to uracil prototrophy, thereby producing strains in which pRS406 sequences and the mutant promoter were integrated between the wild-type promoter and the coding sequence for STE6. To effect the removal of the wild-type promoter and pRS406 sequences, URA colonies were grown overnight in YPD and then streaked out on 5-FOA plates to select for the loss of the URA3 marker. Both the pop-in and pop-out sequence arrangements were confirmed by genomic Southern blotting.

Verification that the point mutations in the STE6 promoter region lead to reduced STE6 transcription was accomplished by performing RNase protection assays on the FY23 derivatives. RNA was extracted from wild-type and STE6 promoter-mutant FY23 cells using the method of Schmitt et al. (1990). The probe for the STE6 transcript was derived from the 385-bp EcoRI$B a m H I$ fragment of STE6, cloned into the polylinker region of pRS406, cut with EcoRI, and transcribed from the T3 promoter. The control transcript utilized was that of SSN6, the probe for which was derived from the SSN6-disrupting plasmid described in the previous section, cut with $A v r I I$, and transcribed from the $\mathrm{T} 7$ promoter. Both probes were produced by in vitro transcription using the Ambion MAXIscript in vitro transcription kit and gel purified. RNase protection assays utilized the Ambion RPAII ribonuclease protection assay kit.

\section{Isolation of nuclei and primer extension analysis} of micrococcal nuclease cleavage sites

Yeast nuclei were isolated using the procedure of Szent-Gyorgyi and Isenberg (1983), with the modifications described by Roth et al. (1992). Micrococcal nuclease digestion of nuclei and subsequent purification of the DNA, as well as nuclease digestion of naked DNA samples, were performed as described previously (Roth et al. 1992), with the micrococcal nuclease concentrations of 0.5 to $5.0 \mathrm{U} / \mathrm{ml}$. The locations of micrococcal nuclease cleavage sites were ascertained by primer extension analysis using Taq polymerase and the primer MS-7 (Shimizu et al. 1991), which corresponds to -338 to -304 of the genomic STE6 sequence. Digested DNA $(\sim 10 \mu \mathrm{g})$ was combined with 0.3 pmole of ${ }^{32} \mathrm{P}$-end-labeled primer in Taq buffer $10 \mathrm{mM}$ Tris- $\mathrm{HCl}$ at $\mathrm{pH} 8.3,50 \mathrm{mM} \mathrm{KCl}, 3 \mathrm{~mm} \mathrm{MgCl}_{2}, 0.05 \% \mathrm{NP}-40,0.05 \%$ Tween 20; Innis et al. 1988) containing $200 \mu \mathrm{M}$ dNTPs; 1 unit of Taq polymerase was added, and the samples were subjected to 25 thermal cycles $\left(94^{\circ} \mathrm{C}\right.$ for $1 \mathrm{~min}, 55^{\circ} \mathrm{C}$ for $2 \mathrm{~min}, 72^{\circ} \mathrm{C}$ for 2 min). After extension, samples were ethanol precipitated and run on $6 \%$ polyacrylamide sequencing gels according to standard procedures (Sambrook et al. 1990).

Isolation of chromatin and analysis of micrococcal nuclease cleavage sites by indirect end labeling

For analysis of plasmid nucleosome positions, chromatin was partially purified by Sepharose chromatography and digested with micrococcal nuclease as described previously (Thoma 1986; Roth et al. 1990), using micrococcal nuclease concentrations of $2-10 \mathrm{U} / \mathrm{ml}$. The DNA was then purified as described previously (Roth et al. 1990), digested with the restriction endonuclease EcoRV, electrophoresed on $1.2 \%$ agarose gels, transferred to nylon (Duralon) membranes, and hybridized to labeled probes by use of the method of Church and Gilbert (1984). The probe used in the current study was the EcoRV-HindIII fragment of TALS (Roth et al. 1990), labeled with $\left[\alpha{ }^{-32} \mathrm{P}\right] \mathrm{dCTP}$ with the Boehringer Mannheim random prime labeling kit.

\section{Analysis of plasmid topoisomer distributions}

For analysis of supercoil density, yeast DNA was rapidly isolated from whole cells by use of the glass bead procedure of Hoffman and Winston (1987). DNA samples were then run on $1.5 \%$ agarose gels containing $40 \mu \mathrm{g} / \mathrm{ml}$ of chloroquine diphosphate for $17-20 \mathrm{hr}$ at $\sim 3 \mathrm{~V} / \mathrm{cm}$, transferred to nylon (Duralon) membranes, and hybridized to labeled probes using the Church and Gilbert (1984) method. The probes employed were linearized derivatives of the TALS or TRPI/ARS1 plasmids, labeled with $\left[\alpha^{-32} \mathrm{P}\right] \mathrm{dCTP}$ with the Boehringer Mannheim random prime labeling kit.

\section{Assay for production of a-factor}

To assay for the generation of a-factor halos, 10-ml cultures of $\alpha$ sst 2 cells (Chan and Otte 1982) were grown overnight. Aliquots $(100 \mu l)$ were then diluted 50- to 100 -fold, spread on YPD plates, and allowed to dry. Strains of interest were spotted over the $\alpha$ sst2 lawns, and the plates were incubated at $30^{\circ} \mathrm{C}$ for $36.48 \mathrm{hr}$.

\section{Assay for $\beta$-galactosidase activity}

$\beta$-Galactosidase assays were performed on crude extracts by use of the method of Rose and Botstein (1983; Rose et al. 1990). Activities were normalized to the amount of protein in the extracts, as determined by the dye-binding assay of Bradford (1976).

\section{Acknowledgments}

We are indebted to Randy Morse for numerous productive discussions and for generous advice on the technical aspects of this work. We thank Mike Kladde, Mike Murphy, Hugh-George Patterton, and Chris Szent-Gyorgyi for helpful and stimulating discussions throughout the course of this study.

The publication costs of this article were defrayed in part by payment of page charges. This article must therefore be hereby marked "advertisement" in accordance with 18 USC section 1734 solely to indicate this fact.

\section{References}

Almer, A. and W. Horz. 1986. Nuclease hypersensitive regions with adjacent positioned nucleosomes mark the gene bound- 
aries of the PHO5/PHO3 locus in yeast. EMBO /. 5: 26812687.

Almer, A., H. Rudolph, A. Hinnen, and W. Horz. 1986. Removal of positioned nucleosomes from the yeast PHO5 promoter upon PHO5 induction releases additional upstream activating DNA elements. EMBO $/$. 5: 2689-2696.

Bradford 1976. A dye binding assay for protein. Anal. Biochem. 72: $248-254$.

Carlson, M., B.C. Osborn, L. Neigeborn, and D. Botstein. 1984. A suppressor of SNF1 mutations causes constitutive highlevel invertase synthesis in yeast. Genetics 107: 19-32.

Chan, R.K. and C.A. Otte. 1982. Isolation and genetic analysis of Saccharomyces cerevisiae mutants supersensitive to G1 arrest by a factor and $\alpha$ factor pheromones. Mol. Cell. Biol. 2: $11-20$.

Chen, S., R.W. West, S.L. Johnson, H. Gans, B. Krueger, and J. Ma. 1993. TSF3, a global regulatory protein that silences transcription of yeast $G A L$ genes also mediates repression by $\alpha 2$ repressor and is identical to SIN4. Mol. Cell. Biol. 11: 6306-6316.

Church, G.M. and W. Gilbert. 1984. Genomic sequencing. Proc. Natl. Acad. Sci. 81: 1991-1995.

Conconi, A., R.M. Widmer, T. Koller, and J. Sogo. 1989. Two different chromatin structures coexist in ribosomal RNA genes throughout the cell cycle. Cell 57: 752-761.

Cordingley, M.G. and G.L. Hager. 1988. Binding of multiple factors to the MMTV promoter in crude and fractionated nuclear extracts. Nucleic Acids Res. 16: 609-628.

Dranginis, A.M. 1986. Regulation of cell type in yeast by the mating type locus. Trends Biochem. Sci. 11: 328-331.

Fong, H.K.W., J.B. Hurley, R.S. Hopokins, R. Miake-Lye, M.S. Johnson, R.F. Doolittle, and M.I. Simon. 1986. Repetitive segmental structure of the transducin beta subunit: Homology with the CDC4 gene and identification of related $\mathrm{mR}$ NAs. Proc. Natl. Acad. Sci. 83: 2162-2166.

Goebl, M. and M. Yanagida. 1991. The TPR snap helix: A novel protein repeat from mitosis to transcription. Trends Biochem. Sci. 16: 173-177.

Grunstein, M. 1990a. Histone function in transcription. Annu. Rev. Cell Biol. 6: 643-678.

- 1990b. Nucleosomes: Regulators of transcription. Trends Genet. 6: 395-400.

Hall, M.N. and A.D. Johnson. 1987. Homeodomain of yeast repressor $\alpha 2$ is a DNA binding domain but is not sufficient for repression. Science 237: 1007-1012.

Herschbach, B.M. and A.D. Johnson. 1993. The yeast $\alpha 2$ protein can repress transcription by RNA polymerases I and II but not III. Mol. Cell. Biol. 13: 4029-4038.

Herskowitz, I. 1989. A regulatory hierarchy for cell specialization in yeast. Nature 342: 749-757.

Hill, J., I.G. Donald, and D.E. Griffiths. 1991. DMSO-enhanced whole cell yeast transformation. Nucleic Acids Res. 19: 5791.

Hirschorn, J.N., S.A. Brown, C.D. Clark, and F. Winston. 1992. Evidence that SNF2/SWI2 and SNF5 activate transcription in yeast by altering chromatin structure. Genes \& Dev. 6: 2288-2298.

Hoffman, C.S. and F. Winston. 1987. A ten-minute DNA preparation from yeast efficiently releases autonomous plasmids for transformation of Escherichia coli. Gene 57: 267-272.

Innis, M.A., K.B. Myambo, D.H. Gelfand, and M.A. Brow. 1988. DNA sequencing with Thermus aquaticus DNA polymerase and direct sequencing of polymerase chain reaction-amplified DNA. Proc. Natl. Acad. Sci. 85: 9436-9440.

Jiang, Y.W. and D.J. Stillman. 1992. Involvement of the SIN4 global transcriptional regulator in the chromatin structure of
Saccharomyces cerevisiae. Mol. Cell. Biol. 12: 4503-4514.

Keleher, C.A., C. Goutte, and A.D. Johnson. 1988. The yeast cell type specific repressor $\alpha 2$ acts cooperatively with a non-cell type specific protein. Cell 53: 927-936.

Keleher, C.A., S. Passmore, and A.D. Johnson. 1989. Yeast repressor $\alpha 2$ binds to its operator cooperatively with yeast protein MCM1. Mol. Cell. Biol. 9: 5228-5230.

Keleher, C.A., M.J. Redd, J. Schultz, M. Carlson, and A.D. Johnson. 1992. Ssn6-Tupl is a general repressor of transcription in yeast. Cell 68: 709-719.

Kronstad, J.W., J.A. Holly, and V.L. MacKay. 1987. A yeast operator overlaps an upstream activation site. Cell 50:369377.

Laughton, A. and M.P. Scott. 1984. The sequence of a Drosophila segmentation gene: Protein structure homology with DNA binding proteins. Nature 310: 25-30.

Matallana, E., L. Franco, and J.E. Perez-Ortin. 1992. Chromatin structure of the yeast SUC2 promoter in regulatory mutants. Mol. Gen. Genet. 231: 395-400.

McDonnell, D.P., E. Vegeto, and B.W. O'Malley. 1992. Identification of a negative regulatory function for steroid receptors. Proc. Natl. Acad. Sci. 89: 10563-10567.

McGrath, J.P. and A. Varshavsky. 1989. The yeast STE6 gene encodes a homologue of the mammalian multidrug resistance P-glycoprotein. Nature 340: 400-404.

Morse, R.H. 1991. Topoisomer heterogeneity of plasmid chromatin in living cells. I. Mol. Biol. 222: 133-137.

- 1993. Nucleosome disruption by transcription factor binding in yeast. Science 262: 1563-1566.

Morse, R.H., S.Y. Roth, and R.T. Simpson. 1992. A transcriptionally active tRNA gene interferes with nucleosome positioning in vivo. Mol. Cell. Biol. 12: 4015-4025.

Mukai, Y., S. Harashima, and Y. Oshima. 1991. AAR1/TUP1 protein, with a structure similar to that of the $\beta$ subunit of $G$ proteins, is required for a $1-\alpha 2$ and $\alpha 2$ repression in cell type control of Saccharomyces cerevisiae. Mol. Cell. Biol. 11: 3773-3779.

Nehlin, J.O., M. Carlberg, and H. Ronne. 1991. Control of yeast GAL genes by MIG1 repressor: A transcriptional cascade in the glucose response. EMBO /. 10: 3373-3377.

Passmore, S., R. Elble, and B.K. Tye. 1989. A protein involved in minichromosome maintenance in yeast binds a transcriptional enhancer conserved in eukaryotes. Genes \& Dev. 3: 921-935.

Perlmann, T. and O. Wrange. 1988. Specific glucocorticoid receptor binding to DNA reconstituted in a nucleosome. EMBO f. 7: 3073-3079.

Prelich, G. and F. Winston. 1993. Mutations that suppress the deletion of an upstream activating sequence in yeast: Involvement of a protein kinase and histone $\mathrm{H} 3$ in repressing transcription in vivo. Genetics 135: 665-676.

Prior, C.P., C.R. Cantor, E.M. Johnson, V.C. Littau, and V.G. Allfrey. 1983. H3 accessibility in transcriptionally active and inactive states of rDNA chromatin. Cell 34: 1033-1042.

Richard-Foy, H. and G.L. Hager. 1987. Sequence-specific positioning of nucleosomes over the steroid-inducible MMTV promoter. EMBO 1. 6: 2321-2328.

Rose, M.D. and D. Botstein. 1983. Construction and use of gene fusions lacZ ( $\beta$-galactosidase) which are expressed in yeast. Methods Enzymol. 101: 167-180.

Rose, M.D., F. Winston, and P. Hieter. 1990. Methods in yeast genetics: A laboratory course manual. Cold Spring Harbor Laboratory Press, Cold Spring Harbor, New York.

Roth, S.Y., A. Dean, and R.T. Simpson. 1990. Yeast $\alpha 2$ repressor positions nucleosomes in TRP1/ARS1 chromatin. Mol. Cell. Biol. 10: $2247-2260$. 
Roth, S.Y., M. Shimizu, L. Johnson, M. Grunstein, and R.T. Simpson. 1992. Stable nucleosome positioning and complete repression by the yeast $\alpha 2$ repressor are disrupted by aminoterminal mutations in histone H4. Genes \& Dev. 6: 411425.

Rothstein, R. 1991. Targeting, disruption, replacement, and allele rescue: Integrative DNA transformation in yeast. Methods Enzymol. 194: 281-301.

Sambrook, J., E.F. Fritsch, and T. Maniatis. 1989. Molecular cloning: A laboratory manual, 2nd ed. Cold Spring Harbor Laboratory Press, Cold Spring Harbor, New York.

Schmid, A., K.-D. Fascher, and W. Horz. 1992. Nucleosome disruption at the yeast $\mathrm{PHO} 5$ promoter upon $\mathrm{PHO} 5$ induction occurs in the absence of DNA replication. Cell 71: 853-864.

Schmitt, M.E., T.A. Brown, and B.L. Trumpower. 1990. A rapid and simple method for preparation of RNA from Saccharomyces cerevisiae. Nucleic Acids Res. 18: 3091-3092.

Schultz, J. and M. Carlson. 1987. Molecular analysis of SSNG, a gene functionally related to the $S N F 1$ protein kinase of Saccharomyces cerevisiae. Mol. Cell. Biol. 7: 3637-3645.

Schultz, J., L. Marshall-Carlson, and M. Carlson. 1990. The $\mathrm{N}$-terminal TPR region is the functional domain of SSNG, a nuclear phosphoprotein of Saccharomyces cerevisiae. Mol. Cell. Biol. 10: 4744-4756.

Shimizu, M., S.Y. Roth, C. Szent-Gyorgyi, and R.T. Simpson. 1991. Nucleosomes are positioned with base pair precision adjacent to the $\alpha 2$ operator in Saccharomyces cerevisiae. EMBO I. 10: 3033-3041.

Sikorski, R.S. and P. Hieter. 1989. A system of shuttle vectors and yeast host strains designed for efficient manipulation of DNA in Saccharomyces cerevisiae. Genetics 122: 19-27.

Sikorski, R.S., M.S. Boguski, M. Goebl, and P. Hieter. 1990. A repeating amino acid motif in CDC23 defines a family of proteins and a new relationship among genes required for mitosis and RNA synthesis. Cell 60: 307-317.

Simpson, R.T. 1990. Nucleosome positioning can affect the function of a cis-acting DNA element in vivo. Nature 343: 387-389.

- 1991. Nucleosome positioning: Occurrence, mechanisms, and functional consequences. Prog. Nucleic Acids Res. Mol. Biol. 40: 143-184.

Simpson, R.T., S.Y. Roth, R.H. Morse, H.-G. Patterton, J.P. Cooper, M. Murphy, M.P. Kladde, and M. Shimizu. 1993. Nucleosome positioning and transcription. Cold Spring Harbor Symp. Quant. Biol. (in press).

Southern, E.M. 1975. Detection of specific sequences among DNA fragments separated by gel electrophoresis. I. Mol. Biol. 98: 503-517.

Szent-Gyorgyi, C. and I. Isenberg. 1983. The organization of oligonucleosomes in yeast. Nucleic Acids Res. 11:37173736.

Thoma, F. 1986. Protein-DNA interactions and nuclease sensitive regions determine nucleosome positions on yeast plasmid chromatin. I. Mol. Biol. 190: 177-190.

Thoma, F., L.W. Bergman, and R.T. Simpson. 1984. Nuclease digestion of circular TRP1/ARS1 chromatin reveals positioned nucleosomes separated by nuclease sensitive regions. J. Mol. Biol. 177: 715-733.

Thrash-Bingham, C. and W.L. Fangman. 1989. A yeast mutation that stabilizes a plasmid bearing a mutated $A R S 1$ element. Mol. Cell. Biol. 9: 809-816.

Trumbly, R.J. 1986. Isolation of Saccharomyces cerevisiae mutants constitutive for invertase synthesis. J. Bacteriol. 166: 1123-1127.

Vidal, M. and R.F. Gaber. 1991. RPD3 encodes a second factor required to achieve maximum positive and negative tran- scriptional states in Saccharomyces cerevisiae. Mol. Cell. Biol. 11: 6317-6327.

Vidal, M., R. Strich, R.E. Esposito, and R.F. Gaber. 1991. RPD1 (SIN3/UME4) is required for maximal activation and repression of diverse yeast genes. Mol. Cell. Biol. 11: 6306-6316.

Williams, F.E. and R.J. Trumbly. 1990. Characterization of TUP1, a mediator of glucose repression in Saccharomyces cerevisiae. Mol. Cell. Biol. 10: 6500-6511.

Williams, F.E., U. Varanasi, and R.J. Trumbly. 1991. The CYC8 and TUP1 proteins involved in glucose repression in Saccharomyces cerevisiae are associated in a protein complex. Mol. Cell. Biol. 11: 3307-3316.

Wilson, K.L. and I. Herskowitz. 1986. Sequences upstream of the STE6 gene required for its expression and regulation by the mating type locus in Saccharomyces cerevisiae. Proc. Natl. Acad. Sci. 83: 2536-2540.

Wolffe, A.P. 1990. New approaches to chromatin function. New Biol. 2: 1-8.

- 1992. Chromatin structure and function. Academic Press, Inc., San Diego, CA.

Zhang, M., L.S. Rosenblum-Vos, C.V. Lowry, K.A. Boakye, and R.S. Zitomer. 1991. A yeast protein with homology to the $\beta$ subunit of G proteins is involved in control of heme-regulated and catabolite-repressed genes. Gene 97: 153-161. 


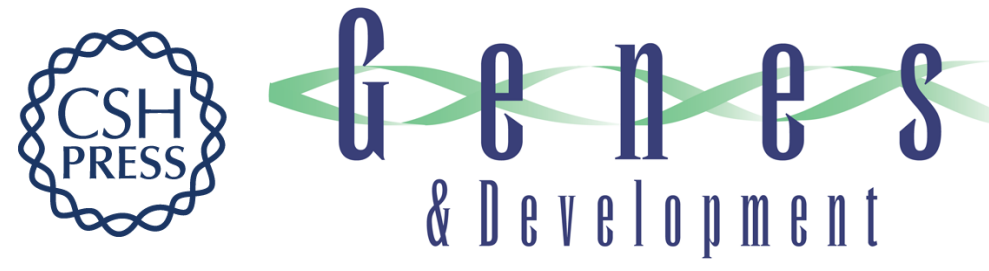

\section{The global transcriptional regulators, SSN6 and TUP1, play distinct roles in the establishment of a repressive chromatin structure.}

J P Cooper, S Y Roth and R T Simpson

Genes Dev. 1994, 8:

Access the most recent version at doi:10.1101/gad.8.12.1400

References This article cites 66 articles, 27 of which can be accessed free at:

http://genesdev.cshlp.org/content/8/12/1400.full.html\#ref-list-1

License

Email Alerting

Service

Receive free email alerts when new articles cite this article - sign up in the box at the top right corner of the article or click here.

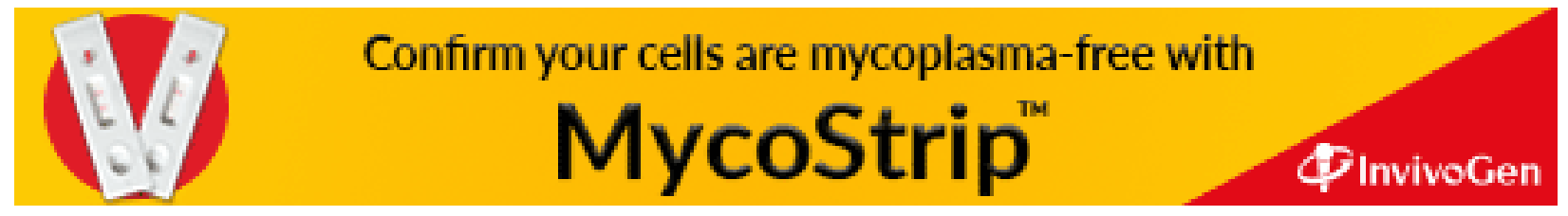

